CLINICAL STUDY

\title{
FSHR gene polymorphisms influence bone mineral density and bone turnover in postmenopausal women
}

\author{
Domenico Rendina, Fernando Gianfrancesco ${ }^{1}$, Gianpaolo De Filippo ${ }^{2}$, Daniela Merlotti ${ }^{3}$, Teresa Esposito ${ }^{1}$, \\ Alessandra Mingione ${ }^{1}$, Ranuccio Nuti ${ }^{3}$, Pasquale Strazzullo, Giuseppe Mossetti and Luigi Gennari ${ }^{3}$ \\ Department of Clinical and Experimental Medicine, Federico II University Medical School, 80131 Naples, Italy, ${ }^{1}$ Human Genetic Diseases Laboratory, \\ Institute of Genetics and Biophysics 'Adriano Buzzati-Traverso', Italian National Research Council, via Pietro Castellino, 111, 80131 Naples, Italy, ${ }^{2}$ Unit \\ of Pediatric Endocrinology, Gaetano Rummo Hospital, 82100 Benevento, Italy and ${ }^{3}$ Department of Internal Medicine, Endocrine-Metabolic Sciences and \\ Biochemistry, University of Siena, 83100 Siena, Italy
}

(Correspondence should be addressed to F Gianfrancesco; Email: gianfrancesco@igb.cnr.it)

\begin{abstract}
Objective: FSH, via its receptor (FSHR), influences bone remodeling and osteoclast proliferation and activity. The aim of this study was to evaluate the influence of two single nucleotide polymorphisms (SNPs) of the FSHR gene on bone mineral density (BMD) and bone turnover markers (bone alkaline phosphatase and type I collagen C-telopeptides) in postmenopausal women.

Methods: Two hundred and eighty-nine unrelated postmenopausal women were genotyped for the SNPs rs1394205 and rs6166. BMD was estimated using dual-energy X-ray absorptiometry and quantitative ultrasound (QUS) methodologies.

Results: AA rs6166 women showed a lower BMD (femoral neck and total body), lower stiffness index (calcaneal QUS), and higher serum levels of bone turnover markers compared to GG rs6166 women. The prevalence of osteoporosis was significantly higher in AA rs6166 women compared with GG rs6166 women. These results were not influenced by circulating levels of FSH and estrogens. Conclusion: The SNP rs6166 of the FSHR gene significantly influences BMD in postmenopausal women. In particular, AA rs6166 women are at increased risk of postmenopausal osteoporosis compared with GG rs6166 women, independently of circulating levels of FSH and estrogens. Previous studies have demonstrated that this SNP influences cell and tissue response to hyperstimulation of FSHR in vivo and in vitro. Our study results appear in agreement with these experimental data and with known biological actions of FSH/FSHR system in bone homeostasis.
\end{abstract}

European Journal of Endocrinology 163 165-172

\section{Introduction}

Bone is in a constant state of dynamic turnover known as bone remodeling (1). At the site of turnover, osteoblasts lay down new bone, whereas osteoclasts resorb existing bone. Each cell type is regulated by a variety of hormonal and local factors $(2,3)$. If the balance between bone formation and bone resorption is lost, i.e. during inflammation or estrogen deficiency, bone integrity can be dramatically affected, and the patient might develop a metabolic bone disorder (1-3). Bone homeostasis is also influenced by the immune system (4). A critical element in the crosstalk between bone and the immune system is the inducible transcription factor nuclear factor- $\kappa \mathrm{B}(\mathrm{NF}-\kappa \mathrm{B})$, which regulates gene expression during both inflammatory and immune responses as well as bone homeostasis and osteoclast differentiation (5). Recent evidences indicate that TSH and FSH, two structurally related glycoprotein hormones synthesized by the anterior pituitary gland, influence bone remodeling (6-9). In particular,
Sun et al. reported that, independently of the murine or human model, FSH is able to stimulate directly osteoclast formation and metabolic activities via its receptor (FSHR) (8). In fact, both the FSHR null mice and the FSH $\beta$ null mice models indicate that FSH may be required for postmenopausal bone loss (8-12). These osteoclastogenic and pro-resorptive actions of FSH on bone are exerted through a regulation of the NF- $\kappa \mathrm{B}$ signaling pathways (8). These experimental evidences were confirmed by recent clinical observations evidencing a role of FSH in bone loss associated with menopausal transition (13-15). Part of the FSH effects on bone cells could also be mediated through the tumor necrosis factor (TNF)- $\alpha$ system (16). Moreover, experimental models in ovariectomized (OVX) mice suggested that FSH and the immune system interact in the regulation of postmenopausal bone loss. FSH regulates T cell expression of CD40L, a co-stimulatory molecule activated by the antigen-presenting cell receptor CD40. The CD40L/CD40 signaling is the pathway by which the OVX-associated increase in FSH regulates 
activation of $\mathrm{T}$ cells and production of TNFs by T cells. Interestingly, this is also the pathway by which T cells regulate stromal cells production of receptor activator of NF- $\mathrm{BB}$ ligand (RANK-L) $(17,18)$. On the basis of these experimental evidences, we planned the present study to evaluate the possible influence of FSHR polymorphisms on bone mineral density (BMD) and bone turnover markers in postmenopausal women.

\section{Patients and methods}

\section{Patients}

The overall sample $(n=289)$ was composed by 192 unrelated postmenopausal women referred to the Department of Internal Medicine, Endocrine-Metabolic Sciences and Biochemistry of the University of Siena, and 97 postmenopausal women evaluated in the Department of Clinical and Experimental Medicine of the Federico II University Medical School in Naples, between 1st January 2007 and 30th June 2009. All subjects were enrolled with the collaboration of the general practitioners who randomly invited postmenopausal women aged between 50 and 75 years in their database to undergo a clinical screening for osteoporosis in these centers. At enrollment, calcium intake, alcohol consumption, and physical activity were evaluated in each patient. Calcium intake and alcohol consumption were assessed by using previously validated semiquantitative diet questionnaires $(18,19)$. In cases of calcium supplementation, the amount of calcium supplemented each day was added to the dietary intake. Total daily calcium intake was expressed as a score ranging from 1 to 3 , according to the following scale: score 1 for a calcium intake lower than $500 \mathrm{mg} /$ day, score 2 for a calcium intake from 500 to $1000 \mathrm{mg} /$ day, and score 3 for an intake higher than $1000 \mathrm{mg} /$ day. Alcohol consumption was also expressed as a score ranging from 1 to 3 (score 1 for an alcohol consumption lower than $1000 \mathrm{mg} /$ day, score 2 for an alcohol consumption from 1000 to $2000 \mathrm{mg} /$ day, and score 3 for an alcohol consumption higher than $2000 \mathrm{mg} /$ day). A semi-quantitative questionnaire was also used to evaluate daily physical activity according to the following scale: score 3 (high physical activity) was assigned to women who exercised regularly; score 2 (moderate physical activity) was assigned to women who did not exercise regularly but participated in daily activities, such as house cleaning, climbing stairs, or walking; score 1 (low physical activity) was assigned to women who did not participate in any of above-mentioned activities $(19,20)$.

For all subjects, a detailed medical history was obtained. In particular, self-reported information on previous fractures was collected through a specific fixed-sequence questionnaire. Only symptomatic, nontraumatic fractures of the hip, vertebral bodies, and the wrist were considered for the purposes of the present study. Women with a history of bone disease other than primary osteoporosis or who had used hormone replacement therapy, bone active drugs, or drugs that could potentially affect bone metabolism were excluded from analysis. None of the women enrolled in this study reported the assumption of vitamin $\mathrm{D}$ and/or calcium supplementation. All subjects were Caucasian and of Italian descent. The study was conducted according to the Declaration of Helsinki, and all enrolled subjects gave written informed consent. The study protocol received the approval of the local ethical committees. General characteristics of the entire cohort are shown in Table 1.

Table 1 Anthropometric data and clinical characteristics of study population classified according to rs 1394205 and rs6166 FSHRgenotypes.

\begin{tabular}{|c|c|c|c|c|c|c|c|c|}
\hline & \multicolumn{4}{|c|}{ rs1394205 } & \multicolumn{4}{|c|}{ rs6166 } \\
\hline & AA & $A G$ & GG & $P$ & AA & $A G$ & GG & $P$ \\
\hline Number & 29 & 117 & 143 & & 81 & 132 & 76 & \\
\hline Age (years) & $63.5 \pm 7.6$ & $63.5 \pm 6.3$ & $64.0 \pm 6.9$ & 0.91 & $63.4 \pm 6.5$ & $63.7 \pm 6.6$ & $64.2 \pm 6.9$ & 0.89 \\
\hline BMl $\left(\mathrm{kg} / \mathrm{m}^{2}\right)$ & $24.7 \pm 3.9$ & $25.5 \pm 4.0$ & $26.1 \pm 4.8$ & 0.43 & $25.9 \pm 5.6$ & $25.6 \pm 3.8$ & $25.7 \pm 4.1$ & 0.67 \\
\hline Pregnancy (number) & $1.7 \pm 0.7$ & $1.6 \pm 1.1$ & $1.4 \pm 0.7$ & 0.58 & $1.5 \pm 0.5$ & $1.6 \pm 0.9$ & $1.4 \pm 0.8$ & 0.61 \\
\hline Age at menarche (years) & $12.8 \pm 1.6$ & $12.7 \pm 1.4$ & $12.9 \pm 1.2$ & 0.47 & $12.8 \pm 1.3$ & $12.8 \pm 1.6$ & $12.9 \pm 1.5$ & 0.54 \\
\hline Age at menopause (years) & $46.8 \pm 6.5$ & $48.9 \pm 4.5$ & $47.9 \pm 7.0$ & 0.49 & $46.8 \pm 7.3$ & $48.7 \pm 4.6$ & $48.8 \pm 10.3$ & 0.21 \\
\hline \multicolumn{9}{|l|}{ Smoking history } \\
\hline Never smoked & $14(48.3)$ & $58(49.6)$ & $71(49.7)$ & & $41(50.6)$ & $66(47.0)$ & $36(47.4)$ & \\
\hline Past smoker & $4(13.8)$ & $17(14.5)$ & 19 (13.3) & 0.98 & $11(13.6)$ & $17(12.9)$ & $12(15.8)$ & 0.98 \\
\hline Current smoker & $11(37.9)$ & $42(35.9)$ & $53(37.0)$ & & $29(35.8)$ & $49(37.1)$ & $28(36.8)$ & \\
\hline Cigarettes smoked ( $n /$ day) & $6.5 \pm 2.8$ & $6.2 \pm 3.4$ & $6.1 \pm 3.2$ & 0.54 & $6.0 \pm 3.5$ & $6.3 \pm 2.9$ & $6.2 \pm 3.4$ & 0.52 \\
\hline Calcium intake score & $1.7 \pm 0.8$ & $1.7 \pm 0.6$ & $1.8 \pm 0.7$ & 0.87 & $1.7 \pm 0.8$ & $1.7 \pm 0.7$ & $1.9 \pm 0.8$ & 0.79 \\
\hline Physical activity score & $1.5 \pm 0.7$ & $1.4 \pm 0.6$ & $1.4 \pm 0.6$ & 0.76 & $1.4 \pm 0.7$ & $1.4 \pm 0.5$ & $1.5 \pm 0.6$ & 0.75 \\
\hline Alcohol intake score & $1.4+0.5$ & $1.2+0.4$ & $1.3+0.4$ & 0.70 & $1.3+0.5$ & $1.3+0.6$ & $1.2+0.5$ & 0.75 \\
\hline
\end{tabular}

Data are expressed as mean \pm s.D. for continual variables and absolute (percentage) values for categorical variables. FSHR, FSH receptor; BMI, body mass index. The $P$ values were determined using the ANOVA for continuous variables and $\chi^{2}$ test for categorical variables. The single nucleotide polymorphism (SNP) rs 1394205 is located in the $5^{\prime}$ region at position -29 within the core promoter region of the FSHR gene, while the SNP rs6166 occurs at codon 680 and results in the amino acid substitution Ser680Asn. Calcium intake score, physical activity score, and alcohol consumption score were evaluated using specific questionnaires previously validated $(19,20)$. 


\section{Bone densitometry and bone ultrasound analysis}

Lumbar $\left(\mathrm{L}_{2}-\mathrm{L}_{4}\right)$, femoral, and total body BMD were assessed by dual-energy X-ray absorptiometry (DXA; Prodigy Oracle, GE, Wakesha, WI, USA) in all the 289 women. The long-term in vitro precision measured using a spine phantom was $0.4 \%$ in Siena and $0.6 \%$ in Naples. The in vivo precision was $0.9 \%$ for lumbar spine and $1.9 \%$ for proximal femur in both centers. Cross calibration studies on the precision of measurements between the two centers were performed. A correction factor was not considered necessary. DXA data were expressed as $\mathrm{g} / \mathrm{cm}^{2}$ and $T$-score. The diagnoses of osteoporosis, osteopenia, and normal BMD were based on the WHO criteria (21). Quantitative ultrasound (QUS) parameters at the calcaneous were also evaluated (Achilles, Lunar, Madison, WI, USA). These included speed of sound (SOS, m/s) and broadband ultrasound attenuation (BUA, $\mathrm{dB} / \mathrm{MHz}$ ). The coefficients of variation were respectively 2.5 for BUA and $0.5 \%$ for SOS. An additional parameter, the stiffness index (SI), was calculated by a linear combination of SOS and BUA $(\mathrm{SI}=(0.67 \times \mathrm{BUA})+(0.28 \times \mathrm{SOS})-420)$. QUS data were expressed as SI and T-score.

\section{Biochemical analyses}

Serum concentrations of calcium, phosphate, bone alkaline phosphatase (Tandem-R Ostase, Beckman Coulter Inc., Fullerton, CA, USA; interassay coefficient of variation $(\mathrm{CV})<8.1 \%)$, serum type I collagen C-telopeptides (CrossLaps RIA, cross-laps, interassay CV $<6.5 \%$ ), estradiol (RIA, Diasorin Diagnostics, Saluggia, Italy; interassay $\mathrm{CV}<7.9 \%$ ), total testosterone (RIA, Diasorin Diagnostics; CV <10.3\%), sex hormone-binding globulin (SHBG RIA, BIOCODE S.A., Liege, Belgium; interassay $\mathrm{CV}<8.0 \%$ ), $\mathrm{FSH}$ (FSH-CTK-4 kit, IRMA Kit, Diasorin Diagnostics; interassay $\mathrm{CV}<8.1 \%$ ), parathyroid hormone (N-tact PTH SP, IRMA Kit, Diasorin Diagnostics; interassay CV $<4.9 \%$ ), and 25-hydroxyvitamin D (250H vitamin D; 125I RIA Kit, Diasorin Diagnostics; interassay CV $<11 \%$ ) were evaluated at baseline. Free androgen and estrogen indexes were calculated as the percent ratios between total hormone levels and SHBG.

\section{Genetic analyses}

The human FSHR gene maps on chromosome 2p21-p16, spanning on a genomic region of about $192 \mathrm{~kb}$ (22). After consulting the HapMap database (http://www.hapmap.org/index.html.en), we identified two main blocks of linkage disequilibrium (LD) along this gene, with few major haplotypes. The first block included the FSHR 5'-upstream region and exons 1-4. The second block included the exons 5-10 and the remaining N-terminal portion of the FSHR gene.
To date, the National Center for Biotechnology Information (NCBI) single nucleotide polymorphism (SNP) database (http://www.ncbi.nlm.nih.gov/SNP/) indicates that there are 1354 known SNPs in the FSHR gene. Only eight of these SNPs are in the coding region, the rest are intronic. One SNP is located in the $5^{\prime}$-untranslated region of FSHR mRNA at position -29 (rs1394205 or ss2189241). The eight SNPs in the coding region (cSNPs) occur at codons 112 (rs1126714), 146 (rs1126715), 307 (rs6165), 309 (rs61743754), 329 (rs6167), 524 (rs12620825), 665 (rs12620825), and 680 (rs6166). Six of these cSNPs are non-synonymous resulting in amino acid substitutions (Asn112Thr, Ala307Thr, Gly309Val/Ala, Ser524Arg, Thr665Ala, and Ser680Asn). While the Ala307Thr (rs6165) and the Ser680Asn (rs6166) polymorphisms are well characterized in terms of frequency and ethnic distribution, there is no detailed information about the prevalence of the other polymorphisms. The two polymorphisms in exon 10 (rs6165 and rs6166) are in LD. Thus, combining information from SNP and HapMap databases, the SNP rs1394205 in the first block and the SNP rs6166 ( $\mathrm{G}>\mathrm{A}$ variation in the second base of codon 680 ) in the second block were selected and genotyped by restriction digestion using the enzymes BseNI and MboII respectively.

\section{Statistical analysis}

The statistical analysis was performed using SPSS (SPSS Inc., Chicago, IL, USA) statistical package (version 11.5). Data in tables are presented as mean \pm s.D. for continuous variables and absolute (percentage) values for discrete variables. ANOVA with Bonferroni correction for multiple comparisons was used to test for between-group differences in continuous variables. For comparison of discrete variables, $\chi^{2}$ test or Fisher's exact test was used. Pearson's correlation coefficient was used to determine relationships between different parameters. All statistical tests were two-sided. A $P$ value below 0.05 was considered as statistically significant.

\section{Results}

The two study groups from Siena and Naples did not significantly differ for age, anthropometric characteristics, biochemical measurements, or prevalence of subjects with osteoporosis and osteopenia. In the overall study population, the absolute (relative) prevalence of FSHR rs1394205 genotypes was 29 (10.0\%), 117 $(40.5 \%)$, and $143(49.5 \%)$ for AA, AG, and GG respectively. Moreover, the absolute (relative) prevalence of FSHR rs6166 genotypes was 81 (28.0\%), 132 $(45.7 \%)$, and $76(26.3 \%)$ for AA, AG, and GG respectively. The observed FSHR allelic frequencies and 
the estimated FSHR haplotype frequencies were consistent with the previously published data from unrelated Caucasian populations $(22,23)$. Moreover, both FSHR SNPs met the Hardy-Weinberg equilibrium $(P=0.45$ and $P=0.14$ for rs1394205 and rs6166 respectively).

Anthropometric data and clinical characteristics of the study population, grouped according to different rs1394205 and rs6166 FSHR genotypes, are reported in Table 1. No significant differences in all parameters evaluated were observed between postmenopausal women classified according to different rs1394205 and rs6166 FSHR genotypes ( $P>0.05$ in all cases).

Biochemical parameters measured in study population, grouped according to different rs1394205 and rs6166 FSHR genotypes, are reported in Table 2. Postmenopausal women with the AA (Asn680/ Asn680) rs6166 genotype showed higher serum levels of bone alkaline phosphatase and type I collagen C-telopeptides compared with those with the GG (Ser680/Ser680) rs6166 FSHR genotype. Other biochemical parameters were not significantly different in the study groups. In particular, serum levels of FSH, SHBG, and free sex hormone were similar in postmenopausal women classified according to different rs1394205 and rs6166 genotypes.

DXA and QUS parameters are reported in Table 3. Postmenopausal women with the AA (Asn680/ Asn680) rs6166 genotype showed a lower femoral neck and total body BMD, expressed as $\mathrm{g} / \mathrm{cm}^{2}$ and T-score, as compared to those with the AG (Ser680/ Asn680) rs6166 and GG (Ser680/Ser680) rs6166 FSHR genotypes. Moreover, heterozygous subjects with AG (Ser680/Asn680) genotype showed significantly lower femoral neck and total body BMD values than subjects with GG (Ser680/Ser680) genotype but significantly higher than subjects with AA (Asn680/ Asn680) genotype. No significant differences were observed in lumbar BMD between subjects with different rs6166 FSHR genotypes, although a trend to lower BMD values was observed in postmenopausal women with AA (Asn680/Asn680) genotype. Conversely, lumbar, femoral neck, and total body BMD were not significantly different in relation to rs1394205 FSHR genotypes.

Similar results were observed when QUS parameters were considered (Table 3). Postmenopausal women with the AA (Asn680/Asn680) rs6166 genotype showed a lower SI (also expressed as T-score) than women with the AG (Ser680/Asn680) and GG (Ser680/Ser680) genotypes. Moreover, heterozygous AG subjects showed significantly lower stiffness values than GG (Ser680/Ser680) subjects but significantly higher than AA (Asn680/Asn680) subjects. Consistent with the DXA parameters, QUS parameters were not significantly different between subjects with different rs1394205 FSHR genotypes. Indeed, a direct linear correlation between total body BMD, femoral neck BMD, and SI was observed in the enrolled subjects (Fig. 1).

The prevalence of osteoporosis, osteopenia, and normal BMD at different sites (lumbar spine, femoral neck, and total body) in relation to FSHR genotypes is reported in Table 4. The prevalence of femoral neck and total body osteoporosis in postmenopausal women with AA (Asn680/Asn680) rs6166 FSHR genotype was significantly higher than in postmenopausal women with GG (Ser680/Ser680) rs6166 FSHR genotype.

A model of multivariate analysis indicated that postmenopausal women with the AA (Asn680/ Asn680) rs6166 FSHR genotype had an overall increased risk of osteoporosis (defined as a T-score $\leq-2.5$ S.D. at either spine or hip (21)) and osteopenia (defined as a $T$-score between -1 and -2.5 S.D. at either spine or hip (21)) compared to postmenopausal women with the GG (Ser680/Ser680) rs6166 FSHR genotype, after correction for all the anthropometric, clinical, and biochemical data reported in Tables 1 and 2 (adjusted odds ratio $(95 \%$ confidence interval $)=1.87$ $(1.18-2.70)$ and $1.75(1.25-2.26)$ for osteoporosis and osteopenia respectively).

Table 2 Biochemical parameters of study population classified according to rs1394205 and rs6166 FSHR genotypes.

\begin{tabular}{|c|c|c|c|c|c|c|c|c|}
\hline & \multicolumn{4}{|c|}{ rs1394205 } & \multicolumn{4}{|c|}{ rs6166 } \\
\hline & AA & $A G$ & GG & $P$ & AA & $A G$ & GG & $P$ \\
\hline FSH (Ul/l) & $38.7 \pm 8.5$ & $42.3 \pm 7.9$ & $38.4 \pm 8.7$ & 0.39 & $37.4 \pm 8.1$ & $43.1 \pm 7.8$ & $37.4 \pm 8.2$ & 0.40 \\
\hline $\operatorname{SHBG}(\mu \mathrm{g} / \mathrm{l})$ & $4.59 \pm 1.95$ & $4.28 \pm 1.71$ & $4.91 \pm 1.97$ & 0.41 & $4.31 \pm 1.83$ & $4.95 \pm 1.94$ & $4.39 \pm 1.51$ & 0.49 \\
\hline 17ß-Estradiol (pmol/l) & $37.4 \pm 8.9$ & $34.2 \pm 12.4$ & $34.6 \pm 13.1$ & 0.51 & $35.5 \pm 11.9$ & $35.7 \pm 17.4$ & $32.2 \pm 15.9$ & 0.43 \\
\hline Calcium $(\mathrm{mmol} / \mathrm{l})$ & $2.33 \pm 0.11$ & $2.32 \pm 0.10$ & $2.33 \pm 0.11$ & 0.94 & $2.32 \pm 0.11$ & $2.33 \pm 0.10$ & $2.32 \pm 0.12$ & 0.94 \\
\hline Phosphate (mmol/l) & $1.10 \pm 0.18$ & $1.10 \pm 0.17$ & $1.11 \pm 0.18$ & 0.87 & $1.11 \pm 0.19$ & $1.11 \pm 0.15$ & $1.09 \pm 0.16$ & 0.68 \\
\hline Creatinine $(\mu \mathrm{mol} / \mathrm{l})$ & $77.8 \pm 17.2$ & $76.9 \pm 10.1$ & $76.0 \pm 14.5$ & 0.71 & $77.7 \pm 17.3$ & $76.1 \pm 14.3$ & $76.1 \pm 13.4$ & 0.57 \\
\hline $\mathrm{iPTH}(\mathrm{pmol} / \mathrm{l})$ & $2.91 \pm 1.19$ & $2.42 \pm 1.11$ & $2.81 \pm 1.25$ & 0.58 & $2.58 \pm 0.76$ & $2.59 \pm 0.98$ & $2.88 \pm 1.05$ & 0.39 \\
\hline $25 \mathrm{OHD}_{3}(\mathrm{nmol} / \mathrm{l})$ & $75.1 \pm 30.2$ & $77.8 \pm 25.9$ & $82.4 \pm 29.8$ & 0.35 & $80.4 \pm 27.4$ & $77.8 \pm 23.3$ & $82.6 \pm 24.2$ & 0.45 \\
\hline bALP $(\mu \mathrm{g} / \mathrm{l})$ & $13.7 \pm 4.2$ & $13.6 \pm 4.2$ & $12.9 \pm 4.3$ & 0.47 & $15.8 \pm 3.9$ & $13.3 \pm 4.3$ & $10.1 \pm 3.6$ & $0.02^{a}$ \\
\hline CTX (nmol/l) & $27.1 \pm 3.1$ & $26.2 \pm 3.2$ & $26.2 \pm 2.8$ & 0.59 & $30.7 \pm 3.4$ & $25.4 \pm 3.4$ & $23.2 \pm 2.9$ & $0.02^{\mathrm{b}}$ \\
\hline
\end{tabular}

Data are expressed as mean \pm s.D. FSHR, FSH receptor; bALP, bone alkaline phosphatase; CTX, serum type I collagen C-telopeptides. The $P$ values were

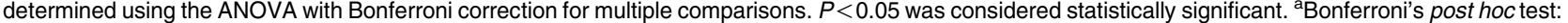
AA versus $G G P=0.03$; AA versus AG $P=0.08$; AG versus $G G P=0.07$. ${ }^{\text {b } B o n f e r r o n i ' s ~ p o s t ~ h o c ~ t e s t: ~ A A ~ v e r s u s ~} G G \quad P=0.02$; AA versus AG $P=0.06$; AG versus $G G P=0.09$. 
Table 3 DXA and QUS parameters of study population classified according to rs1394205 and rs6166 FSHR genotypes.

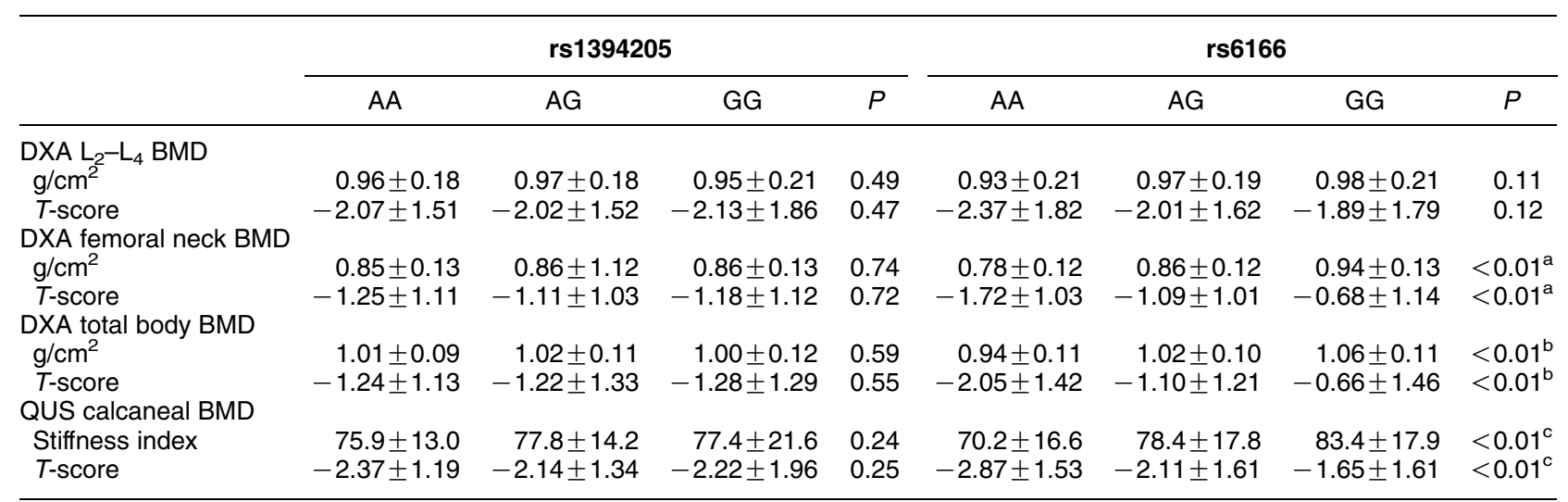

Data are expressed as mean \pm s.D. DXA, dual-energy X-ray absorptiometry (Prodigy Oracle, GE, USA); QUS, quantitative ultrasound (Achilles, Lunar, Madison, WI, USA); FSHR, FSH receptor; BMD, bone mineral density. The stiffness index (SI) was calculated by a linear combination of speed of sound (SOS, $\mathrm{m} / \mathrm{s}$ ) and broadband ultrasound attenuation (BUA, dB/MHz) according to the formula $(\mathrm{SI}=(0.67 \times \mathrm{BUA})+(0.28 \times \mathrm{SOS})-420)$. The $P$ values were determined using the ANOVA. $P<0.05$ was considered statistically significant. ${ }^{2}$ Bonferroni's post hoc test: AA versus $G G P<0.01 ; A A$ versus $A G P=0.02 ;$ AG versus $G G$

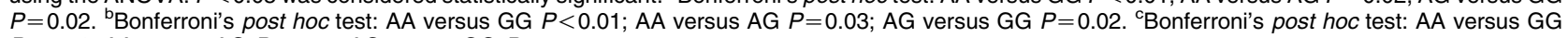
$P<0.01$; AA versus AG $P=0.03 ;$ AG versus $G G=0.03$.

Finally, analyzing the data collected at enrollment, we found 7 patients reporting previous distal forearm fractures, 11 with previous hip fractures, and 15 with symptomatic vertebral fractures. To maximize the power of the statistical analyses, these fractures were considered altogether as a unique composite set (i.e. bone fractures). A trend to increased prevalence of fractures was observed in postmenopausal women with AA (Asn680/Asn680) rs6166 FSHR genotype than in the other genotypes. In fact, $14 / 81$ women $(17.3 \%)$ with AA genotype reported the occurrence of one clinical fracture. Conversely, fractures were observed in 13/81 (9.8\%) women with AG (Asn680/Ser680) genotype and 6/76 (7.9\%) women with GG genotype $(P=0.063)$. No significant differences were observed in the prevalence of fractures between subjects with different rs1394205 FSHR genotypes.

\section{Discussion}

Genetic factors play an important role in the pathogenesis of osteoporosis and fractures (24-27). In fact, despite osteoporosis being a multifactorial disorder, up to $80 \%$ of the variance in BMD is genetically determined, and an interaction between genetic and environmental factors is probably required for the complete clinical expressiveness of the disorder. During previous years, various genetic loci and candidate genes have been associated with the predisposition of osteoporosis and fractures (26). Interestingly, different previous linkage or genome-wide association studies reported an association between BMD at different sites and chromosomal markers strictly related to the 2p21-p16 locus, containing FSHR $(24,28-30)$. Our study results indicate that the polymorphism rs6166 in exon 10 of the FSHR gene significantly influences femoral and total body BMD in postmenopausal women, and that the AA (Asn680/Asn680) allele is associated with an increased risk of osteoporosis and osteopenia. These results do not seem to be influenced by circulating levels of FSH and free estrogens, likely due to the absence of significant variations in such levels in our study groups, which appeared to be homogenous in terms of postmenopausal

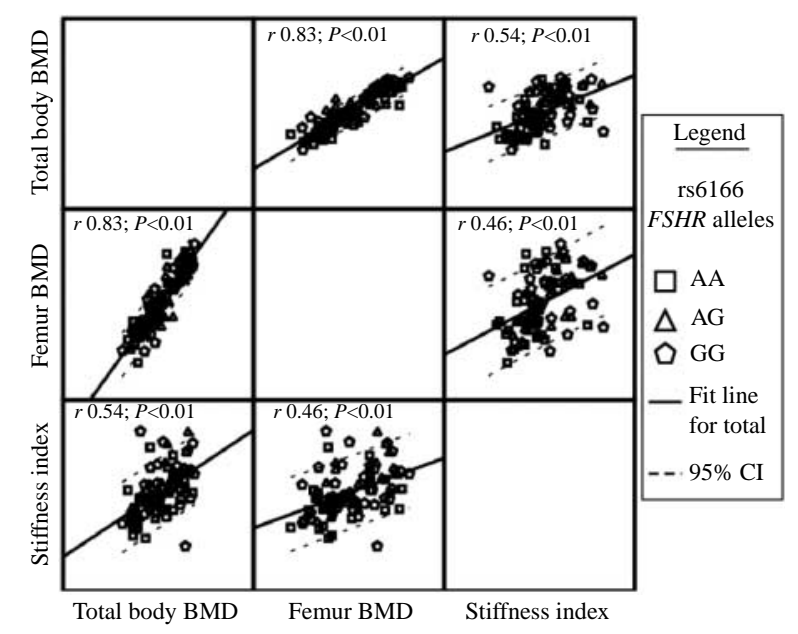

Figure 1 Correlation between total body BMD, femoral neck BMD, and stiffness index in the study population. BMD, bone mineral density; femur, femoral neck. Total body and femoral neck BMD, expressed as $\mathrm{g} / \mathrm{cm}^{2}$, were evaluated using dual-energy X-ray absorptiometry (DXA). Stiffness index (SI) was determined by quantitative ultrasound (QUS) and was calculated by a linear combination of speed of sound (SOS, $\mathrm{m} / \mathrm{s}$ ) and broadband ultrasound attenuation (BUA, $\mathrm{dB} / \mathrm{MHz}$ ) according to the formula $(\mathrm{SI}=(0.67 \times \mathrm{BUA})+(0.28 \times \mathrm{SOS})-420)$. Postmenopausal women were classified according to single nucleotide polymorphism (SNP) rs6166, which occurs at codon 680 of the FSHR gene and causes an amino acid substitution Ser680Asn. Pearson's correlation coefficient was used to determine relationships between different parameters. 
Table 4 Prevalence of normal BMD, osteopenia, and osteoporosis in the study population classified according to rs 1394205 and rs 6166 FSHR genotypes. Data are expressed as absolute (percentage) values.

\begin{tabular}{|c|c|c|c|c|c|c|c|c|}
\hline & \multicolumn{4}{|c|}{ FSHR rs1394205 } & \multicolumn{4}{|c|}{ FSHR rs6166 } \\
\hline & AA (29) & AG (117) & GG (143) & $P$ & $\mathrm{AA}(81)$ & AG (132) & GG (76) & $P$ \\
\hline \multicolumn{9}{|l|}{$\mathrm{L}_{2}-\mathrm{L}_{4} \mathrm{BMD}$} \\
\hline Normal & $11(37.9)$ & $41(35.0)$ & 47 (32.9) & & $22(27.2)$ & $48(36.4)$ & 29 (38.2) & \\
\hline Osteopenia & $4(13.8)$ & $13(11.1)$ & $17(11.9)$ & 0.53 & $10(12.4)$ & $14(10.6)$ & 10 (13.2) & 0.12 \\
\hline Osteoporosis & $14(48.3)$ & $63(53.8)$ & 79 (55.2) & & $49(60.5)$ & $70(53.0)$ & 37 (48.7) & \\
\hline \multicolumn{9}{|c|}{ Femoral neck BMD } \\
\hline Normal & $12(41.4)$ & $47(40.2)$ & $55(38.5)$ & & $21(25.9)$ & $55(41.6)$ & $38(50.0)$ & \\
\hline Osteopenia & $11(37.9)$ & $45(38.5)$ & $53(37.1)$ & 0.57 & $25(30.9)$ & $58(43.9)$ & $26(34.2)$ & $<0.01$ \\
\hline Osteoporosis & $6(20.7)$ & $25(21.4)$ & $35(24.5)$ & & $35(43.2)$ & $19(14.4)$ & $12(15.8)$ & \\
\hline \multicolumn{9}{|c|}{ Total body BMD } \\
\hline Normal & $11(37.9)$ & $48(41.0)$ & $56(39.2)$ & & $22(27.2)$ & $47(35.6)$ & $46(60.5)$ & \\
\hline Osteopenia & $12(41.4)$ & 46 (39.3) & $53(37.0)$ & 0.68 & $24(29.6)$ & $70(53.0)$ & $17(22.4)$ & $<0.01$ \\
\hline Osteoporosis & $6(20.7)$ & $23(19.7)$ & $34(23.8)$ & & $35(43.2)$ & $15(11.4)$ & $13(17.1)$ & \\
\hline
\end{tabular}

FSHR, FSH receptor; BMD, bone mineral density. The diagnosis of osteoporosis, osteopenia, and normal BMD was based on the WHO criteria (21). The $P$ values were determined using the $\chi^{2}$ test for categorical variables. $P<0.05$ was considered statistically significant.

hormonal profile. In particular, serum levels of FSH, estradiol, and SHBG, a regulator of bioavailability of sex steroids (31), were comparable in postmenopausal women with different rs6166 FSHR alleles.

For several years, the stimulation of ovarian folliculogenesis and estrogen production was considered the only function of FSH. More recently, Sun et al. have demonstrated the osteoclastogenic and pro-resorptive actions of FSH (8). FSH exerts these biological actions through its receptor, FSHR, which has been identified on both human and mouse osteoclasts and osteoclast precursors (32). FSH enhances the phosphorylation of downstream RANK-L-sensitive kinases, namely Erk and Akt, as well as of Ik-B $\alpha$, an inhibitor for NF-kB. All of these pathways are known to transduce the proresorptive effects of RANK-L (8). Beside the documented direct effects of FSH on osteoclast signaling pathways, FSH also stimulates the production of TNF- $\alpha$ from bone marrow precursor macrophages (16). This cytokine, which acts as a paracrine factor in bone tissue microenvironment, directly regulates biological activities of osteoclasts via the NF- $\kappa \mathrm{B}$ signaling pathway $(4,5)$, which has been involved in the pathogenesis of metabolic bone disorders including osteoporosis (27).

The FSHR belongs to the large family of G-proteincoupled receptors and comprises three distinct domains: a large extracellular ligand-binding domain, a transmembrane domain consisting of seven membrane-spanning regions with six loops, and an intracellular $\mathrm{COOH}$ terminal tail (32). The FSHR gene has been mapped to chromosome 2p21-p16 and consists of ten exons and nine introns. The first nine exons encode the extracellular domain of the receptor, whereas exon 10 of FSHR encodes the C-terminal end of the extracellular domain, the entire transmembrane domain, and the intracellular domain that is fundamental for signal transduction. Residue 680 is located in the intracellular, C-terminal domain of the receptor, in a region which is highly variable among the three glycoprotein hormone receptors (FSHR, LH receptor, and TSH receptor) $(32,33)$. To date, the functional role of residue 680 has yet to be characterized, but studies in women with normal ovarian function demonstrated that this SNP in exon 10 modulates FSHR function and the cellular response to FSH administration. Interestingly, women with the Ser680/Ser680 variant of the receptor appear to be 'more resistant' to FSH action than women with the other allelic variants, requiring stronger stimulation to obtain the same response $(22$, $23,34-36)$. These differences in ovarian response of women with the 'resistant' variant of the FSHR are subtle and do not impair the capacity of their follicles to ovulate. However, only in the case of pharmaceutical stimulation does the difference become clinically evident $(22,23,32-37)$. Interestingly, the hormonal profile of postmenopausal women is characterized by increased FSH serum levels which chronically stimulate FSHresponsive tissues, including bone, with a greater extent than in premenopausal women. Thus, it is likely that such increased levels of FSH justify the absence of interaction between FSH levels and polymorphic FSHR variants. Moreover, free estradiol levels were reduced and consistent with menopausal levels in all women, without significant differences in relation to FSHR genotype. This implies a direct role of FSHR variants in bone. It can be supposed that, as in other cells expressing FSHR and also in bone cells, the response to FSH is significantly influenced by rs6166 FSHR alleles in condition of chronically increased FSH levels. The molecular mechanism underlying the status of relative 'resistance' of Ser680/Ser680-FSHR variant to FSH is unclear, but the Ser680 residue, which is a potential site of phosphorylation, is located in a region that is important for receptor recycling (38). It should be considered that, in vivo, receptors are usually activated at a low level of occupancy (32). If there is less Ser680-FSHR at the cell surface, both promoter activity and intracellular fate of the receptor protein 
might play a role in determining a quantitatively lower stimulation in response to $\mathrm{FSH}$, but this is difficult to assess in vitro. However, mice with partial loss of Fsh-r gene develop a phenotype similar to that observed in null Fsh-r knockout mice, even though, in the former case, the development of this peculiar phenotype is age dependent. This observation suggests that a partial loss in the cell surface expression of FSHR may be associated with an age-dependent occurrence of related biological changes (33).

In our study population, we observed a discordance between BMD values measured at lumbar spine, femoral neck, and total body BMD, in relation to FSHR genotypes. This could be secondary to concomitant diseases. Common examples observed in the elderly include vertebral osteophytosis, vertebral end plate and facet sclerosis, osteochondrosis, and aortic calcification, each of which could invalidate the BMD measurement at lumbar spine (39). In addition, a physiologic discordance between different skeletal sites could be related to the skeleton's natural adaptive reaction to physiological exogenous and endogenous influencing factors (39). Mechanical strain especially related to weight bearing plays a key role in this kind of discordance (40). Moreover, trabecular sites (such the lumbar spine) are known to have a more rapid rate of bone loss during the early postmenopause than cortical sites (like the proximal femur) (39-41).

The major limitation of our study is the relatively small sample size and its cross-sectional design that has allowed us to only evaluate symptomatic fractures and not incident fractures. However, the selected population was well characterized with the assessment of different variables affecting skeletal health (including calciotropic hormones and sex hormones), and bone mass was examined using two different diagnostic methodologies (DXA and QUS). Moreover, two different regions of FSHR gene were contextually evaluated, including the Ser680Asn variant (rs6166), previously associated with functional differences of the receptor $(22,23$, 34-36). Taken together, both densitometric and biochemical data indicate, for the first time, a significant association between the AA (Asn680/Asn680) rs6166 allele of the FSHR gene and an increased risk of postmenopausal osteoporosis and osteopenia that appears independent of free estrogens or FSH levels. Considering that the different rs6166 FSHR alleles significantly influence the response of ovarian cells to FSH hyperstimulation, further studies are needed to evaluate the biological properties of rs6166 FSHR alleles in bone lineage cells, and particularly in osteoclasts and their precursors.

\section{Declaration of interest}

The authors declare that there is no conflict of interest that could be perceived as prejudicing the impartiality of the research reported.

\section{Funding}

This was supported by an unrestricted grant from Stroder/SIOMMMS (to RD) and ESCEO-AMGEN Fellowship (to D Merlotti).

\section{Acknowledgements}

The authors are grateful to Pasquale and Italiacornelia Rendina for their statistical advice.

\section{References}

1 Karsenty G. The complexities of skeletal biology. Nature $2003 \mathbf{4 2 3}$ 316-318.

2 Boyle WJ, Simonet WS \& Lacey DL. Osteoclast differentiation and activation. Nature $2003 \mathbf{4 2 3} 337-342$.

3 Harada S \& Rodan GA. Control of osteoblast function and regulation of bone mass. Nature 2003423 349-355.

4 Lorenzo J, Horowitz M \& Choi Y. Osteoimmunology: interactions of the bone and immune system. Endocrine Reviews 200829 403-440.

5 Jimi E \& Ghosh S. Role of nuclear factor-kappaB in the immune system and bone. Immunological Reviews 2005208 80-87.

6 Blair HC \& Zaidi M. Osteoclastic differentiation and function regulated by old and new pathways. Reviews in Endocrine $\mathcal{E}$ Metabolic Disorders 20067 23-32.

7 Abe E, Marians RC, Yu W, Wu XB, Ando T, Li Y, Iqbal J, Eldeiry L, Rajendren G, Blair HC, Davies TF \& Zaidi M. TSH is a negative regulator of skeletal remodeling. Cell 2003115 151-162.

8 Sun L, Peng Y, Sharrow AC, Iqbal J, Zhang Z, Papachristou DJ, Zaidi S, Zhu LL, Yaroslavskiy BB, Zhou H, Zallone A, Sairam MR, Kumar TR, Bo W, Braun J, Cardoso-Landa L, Schaffler MB, Moonga BS, Blair HC \& Zaidi M. FSH directly regulates bone mass. Cell 2006125 247-260.

9 Zaidi M, Blair HC, Iqbal J, Zhu LL, Kumar TR, Zallone A \& Sun L. Proresorptive actions of FSH and bone loss. Annals of the New York Academy of Sciences $20071116376-382$.

10 Dierich A. Sairam MR, Monaco L, Fimia GM, Gansmuller A, LeMeur M \& Sassone-Corsi P. Impairing follicle-stimulating hormone (FSH) signaling in vivo: targeted disruption of the FSH receptor leads to aberrant gametogenesis and hormonal imbalance. PNAS 199895 13612-13617.

11 Danilovich N, Babu PS, Xing W, Gerdes M, Krishnamurthy H \& Sairam MR. Estrogen deficiency, obesity, and skeletal abnormalities in follicle-stimulating hormone receptor knockout (FORKO) female mice. Endocrinology 2000141 4295-4308.

12 Kumar TR. FSHbeta knockout mouse model: a decade ago and into the future. Endocrine 200936 1-5.

13 Sowers MR, Greendale GA, Bondarenko I, Finkelstein IS, Cauley JA, Neer RM \& Ettinger B. Endogenous hormones and bone turnover markers in pre- and perimenopausal women: SWAN. Osteoporosis International 200314 191-197.

14 Sowers MR, Jannausch M, McConnell D, Little R, Greendale GA, Finkelstein JS, Neer RM, Johnston J \& Ettinger B. Hormone predictors of bone mineral density changes during the menopausal transition. Journal of Clinical Endocrinology and Metabolism 2006 91 1261-1267.

15 Devleta B, Adem B \& Senada S. Hypergonadotropic amenorrhea and bone density: new approach to an old problem. Journal of Bone and Mineral Metabolism 200422 360-364.

16 Iqbal J, Sun L, Kumar TR, Blair HC \& Zaidi M. Follicle-stimulating hormone stimulates TNF production from immune cells to enhance osteoblast and osteoclast formation. PNAS 2006103 14925-14930.

17 Li JY, Baek KH, Bedi B, Adam J, Yang X, Weitzman M \& Pacifici R. CD4OL mediated $\mathrm{T}$ cell activation is required for estrogen deficiency to induce bone loss. Proceeding of the 31st Annual Meeting of the American Society for Bone and Mineral Research Denver, CO, Journal of Bone and Mineral Research 200924 S64. 
18 Pacifici R. Immune system, FSH and regulation of bone mass. Proceeding of the 10st Annual Meeting of the Società Italiana dell'Osteoporosi del Metabolismo Minerale e delle Malattie dello Scheletro, Torino, Clinical Cases in Mineral and Bone Metabolism 20096 S32.

19 Palomba S, Numis FG, Mossetti G, Rendina D, Vuotto P, Russo T, Zullo F, Nappi C \& Nunziata V. Effectiveness of alendronate treatment in postmenopausal women with osteoporosis: relationship with BsmI vitamin D receptor genotypes. Clinical Endocrinology $2003 \mathbf{5 8} 365-371$.

20 Palomba S, Numis FG, Mossetti G, Rendina D, Vuotto P, Russo T, Zullo F, Nappi C \& Nunziata V. Raloxifene administration in postmenopausal women with osteoporosis: effect of different BsmI vitamin D receptor genotypes. Human Reproduction 2003 18 192-198.

21 Osteoporosis Prevention, Diagnosis, and Therapy Consensus Statement 2000. Journal of the American Medical Association 2001285 785-795.

22 Sudo S, Kudo M, Wada S, Sato O, Hsueh AJ \& Fujimoto S. Genetic and functional analyses of polymorphisms in the human FSH receptor gene. Molecular Human Reproduction 2002 8 893-899.

23 Gromoll J \& Simoni M. Genetic complexity of FSH receptor function. Trends in Endocrinology and Metabolism 200516 368-373.

24 Rivadeneira F, Styrkársdottir U, Estrada K, Halldórsson BV, Hsu YH, Richards JB, Zillikens MC, Kavvoura FK, Amin N, Aulchenko YS, Cupples LA, Deloukas P, Demissie S, Grundberg E, Hofman A, Kong A, Karasik D, van Meurs JB, Oostra B, Pastinen T, Pols HA, Sigurdsson G, Soranzo N, Thorleifsson G, Thorsteinsdottir U, Williams FM, Wilson SG, Zhou Y, Ralston SH, van Duijn CM, Spector T, Kiel DP, Stefansson K, Ioannidis JP \& Uitterlinden AG. Genetic Factors for Osteoporosis (GEFOS) Consortium. Twenty bone-mineral-density loci identified by large-scale meta-analysis of genome-wide association studies. Nature Genetics 200941 1199-1206.

25 Ferrari S. Human genetics of osteoporosis. Best Practice \& Research. Clinical Endocrinology \& Metabolism 20082 723-735.

26 Eisman JA. Genetics of osteoporosis. Endocrine Reviews 199920 788-804.

27 Raisz LG. Pathogenesis of osteoporosis: concepts, conflicts, and prospects. Journal of Clinical Investigation 2005115 3318-3325.

28 Demissie S, Dupuis J, Cupples LA, Beck TJ, Kiel DP \& Karasik D. Proximal hip geometry is linked to several chromosomal regions: genome-wide linkage results from the Framingham Osteoporosis Study. Bone $200740743-750$.

29 Niu T, Chen C, Cordell H, Yang J, Wang B, Wang Z, Fang Z, Schork NJ, Rosen CJ \& Xu X. A genome-wide scan for loci linked to forearm bone mineral density. Human Genetics 1999104 226-233.

30 Styrkarsdottir U, Halldorsson BV, Gretarsdottir S, Gudbjartsson DF, Walters GB, Ingvarsson T, Jonsdottir T, Saemundsdottir J, Center JR, Nguyen TV, Bagger Y, Gulcher JR, Eisman JA,
Christiansen C, Sigurdsson G, Kong A, Thorsteinsdottir U \& Stefansson K. Multiple genetic loci for bone mineral density and fractures. New England Journal of Medicine 2008358 2355-2365.

31 Caldwell JD \& Jirikowski GE. Sex hormone binding globulin and aging. Hormone and Metabolic Research 200941 173-182.

32 Meduri G, Bachelot A, Cocca MP, Vasseur C, Rodien P, Kuttenn F, Touraine P \& Misrahi M. Molecular pathology of the FSH receptor: new insights into FSH physiology. Molecular and Cellular Endocrinology 2008282 130-142.

33 Danilovich N \& Sairam MR. Targeting gonadotropin receptor genes: reproductive biology, aging, and related health implications. Endocrine 200526 219-226.

34 Perez Mayorga M, Gromoll J, Behre HM, Gassner C, Nieschlag E \& Simoni M. Ovarian response to follicle-stimulating hormone (FSH) stimulation depends on the FSH receptor genotype. Journal of Clinical Endocrinology and Metabolism 200085 3365-3369.

35 de Castro F, Ruiz R, Montoro L, Pérez-Hernández D, Sánchez-Casas Padilla E, Real LM \& Ruiz A. Role of follicle-stimulating hormone receptor Ser680Asn polymorphism in the efficacy of folliclestimulating hormone. Fertility and Sterility 200380 571-576.

36 de Castro F, Morón FJ, Montoro L, Galán JJ, Hernández DP, Padilla ES, Ramírez-Lorca R, Real LM \& Ruiz A. Human controlled ovarian hyperstimulation outcome is a polygenic trait. Pharmacogenetics $2004 \mathbf{1 4} 285-293$.

37 Behre HM, Greb RR, Mempel A, Sonntag B, Kiesel L, Kaltwasser P, Seliger E, Röpke F, Gromoll J, Nieschlag E \& Simoni M. Significance of a common single nucleotide polymorphism in exon 10 of the follicle-stimulating hormone (FSH) receptor gene for the ovarian response to FSH: a pharmacogenetic approach to controlled ovarian hyperstimulation. Pharmacogenetics and Genomics 2005 15 451-456.

38 Krishnamurthy H, Kishi H, Shi M, Galet C, Bhaskaran RS, Hirakawa T \& Ascoli M. Postendocytotic trafficking of the follicle-stimulating hormone (FSH)-FSH receptor complex. Molecular Endocrinology 200317 2162-2176.

39 El Maghraoui A \& Roux C. DXA scanning in clinical practice. Quarterly Journal of Medicine 2008101 605-617.

40 El Maghraoui A, Mouinga Abayi DA, Ghozlani I, Mounach A, Nouijai A, Ghazi M, Achemlal L \& Bezza A. Prevalence and risk factors of discordance in diagnosis of osteoporosis using spine and hip bone densitometry. Annals of the Rheumatic Diseases 200766 271-272.

41 Blank RD, Malone DG, Christian RC, Vallarta-Ast NL, Krueger DC, Drezner MK, Binkley NC \& Hansen KE. Patient variables impact lumbar spine dual energy X-ray absorptiometry precision. Osteoporosis International $2006 \mathbf{1 7} 768-774$.

Received 27 February 2010

Accepted 24 March 2010 